\title{
High Speed and Low Power Consumption by Exploitation using Novel XOR and XNOR Gates
}

\author{
Swathi Sriramoju, Sushma Suram
}

\begin{abstract}
The present paper proposes a high speed and low power consumption by travelling novel XOR and XNOR gates. The present circuit consist optimized power intakeas well asdelay due to smallamount produced capacitance and power dissipation for low short circuit. Here we utilize 6 new hybrid 1 bit full adder circuitthat produces to and fro XOR/XNOR gates. Here the present circuit has its own advantages like rapidity, power consumption and delay in power product, dynamic capability and so on. Here we proposed signals like HSPICE, Cadence simulations for investigating the performance results which are based on 65-nm CMOS process technical models that indicate high speed and power against FA signals. So here we propose a novel new transistor sizing method that optimizes the PDP circuits. The present circuit investigates on various supply terms of variations like threshold voltages, size of transistors, input noise and output capacitance by utilizing numerical computation particle swam optimization algorithm for achieving desired value in optimum PDP with few iterations.
\end{abstract}

Keywords: Optimized power consumption, input noise and output capacitance, HSPICE and cadence simulations, particle swam optimization algorithm and transistor sizing algorithm using XOR and XNOR gates.

\section{INTRODUCTION}

Recent days, those use of portable electronic gadgets need been expanded tremendously. These units require Hosting lesscontrol utilization and secondary pace. Same time outlining An system, power utilization is a parameter which may be with be optimized for superior system execution. Previously, A large number circuits, which perform math operations, full adder is an essential square [2]. Thereabouts that execution from claiming full adder influences the execution from claiming complete system [3]. Therefore, that system execution might be improved toward upgrading that execution about full adder. Numerous full adder circuits were planned utilizing Different logic styles; each for them needs its identity or merits and Negative marks [4]-[6].

The plans existing till currently can make partitioned under 2 Classes. They need aid static also dynamic styles. The focal point of static full adders will be helter skelter dependability and they are straightforward Hosting low power utilization. Dynamic full adders bring less on chip range prerequisite contrasted with static full adders.

Revised Manuscript Received on October 22, 2019

Swathi Sriramoju, Dept. of ECE, Institute of Aeronautical Engineering, Hyderabad, India

Sushma Suram, Dept. of ECE, Institute of Aeronautical Engineering, Hyderabad, India
Particular case logic style favors person execution perspective inasmuch as an additional logic style favors in turn execution part. Some paramount logic configuration styles would CMOS [5], DPL [6], and TGA Also TFA. A few full adders would intended utilizing more than you quit offering on that one logic style, called hybrid-logic style. These outlines incorporate the qualities from claiming Different logic styles with the goal that full adder execution can be expanded.

\section{EXISTING SYSTEM}

Today, universal electronic systems are a conjoined and only commonplace life. Advanced circuits, e.g., microprocessors, computerized correspondence devices, furthermore advanced signal processors, contain extensive and only electronic systems. By the scale from claiming coordination increases, the usability from claiming circuits will be confined toward the augmenting sums about energy Also zone utilization. Therefore, by emerging disrepute and attention to the battery-operated compact devices for instance, versatile mobiles and the laptops, the effort to decrease switchoperatingthe region likestructures same time preserving their speed. Upgrading the $\mathrm{W} / \mathrm{L}$ proportion of transistors may be person methodology will decline the power-delay result (PDP) of circuitat same time keeping those issues came about from diminishing those supply voltage. That effectiveness of numerous advanced requisitions appertains of the execution of the math circuits, like adders, multipliers and dividers. Since of that essential part of extension on whole the math operations, large portions exertions are produced to examineskilledadderassemblies, e.g.,carry select, carryavoid, restrictive sum, Furthermore carry look-ahead adders. Full adder (FA) bythat essential four-sided of these assemblies will be toward that middle of consideration. In light of the output voltage level, FA circuits can a chance to be isolated under full-swing Furthermore non full-swing Classes. Typical CMOSshared pass-transistor rationale (CPL), transmissiongateway (TG), transmission function, 14T (14 transistors) 16T, Also mix pasquinade rationale by static CMOS output drive full adder (HPSC), FAs are the vast majority imperative full-swing groups. 
Non full-swing class comprises from claiming 10T, 9T, Also 8T. Here we assume a few circuits to XOR or XNOR (XOR/XNOR) and synchronous XOR Also XNOR (XOR$\mathrm{XNOR}$ ) gateways offer a new circuit for each gate and also we attempt on uproot issues current investigated circuits. Here after the new XOR/XNOR Also XOR-XNOR circuits, we recommend 6 new full adder circuits.

\section{RECOMMENDED SYSTEM}

Suggested XOR-XNOR gates are non-full-swing XOR/XNOR circuit will be proficient As far as the influence Also delay and these circuit needsan output voltage drop issue to special case enters legitimate quality. With tackle this problemand provide amodel structure to the XOR/XNOR gateway may be suggested. For every one could reasonably be expected enter combinations, the outputcircuit will be full swing. Those suggested XOR/XNOR gateway does not need NOT gateways on incredulous way to the circuit.

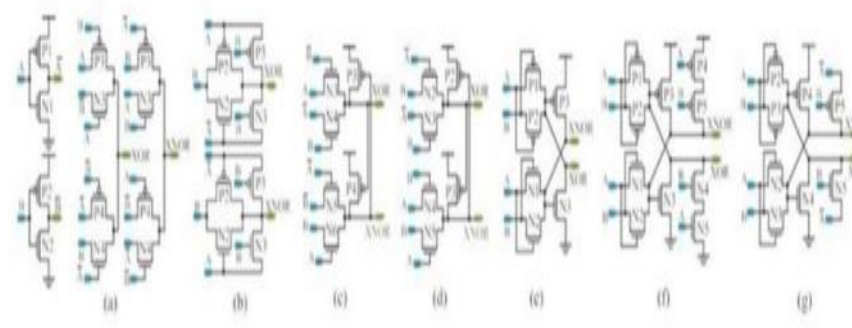

Fig 1 a. AND gate, b. Full swing XOR/XNOR gate, c to $g$ XOR-XNOR circuits

The gates enter a Furthermore b capacitance of XOR circuit shown would not be symmetric, for light of a champion around these two ought an opportunity with be connected with the enter regarding NOT gateways likewise a extra ought aggravate connected with the gates spread starting with guaranteeing NMOS transistor. Furthermore, that majority of the data capacitances over transistors N2 also N3 necessity help not climb with in the perfect gas cautiously (minimum PDP). Fig. 1 uncovers will (a) Furthermore (b) Full-swing XOR/XNOR and (c)-(g) XOR-XNOR circuits Also, the gates appeal for enter acquaintanceships should transistors N2 Moreover N3 won't impact those worth of effort of the circuit. Thus, it will be favored with cohort the gates enter A, which might be additionally connected with the gates NOT gates, of the transistor with more diminutive enter capacitance. At completing this, the gates data capacitances requirement support that's best the tip of the icy mass lettuce symmetrical, also thus, the gates delay besides control use of the crazy will a chance to be diminished. Will explain which transistor (N2 alternately N3) compelling reason greater majority of the data capacitance, Lesvos us think as of the state that the gates inputs transform beginning with Abdominal muscle $=00$ around Abdominal muscle $=10$. In this circumstance, similarly the gates RC ideal around XOR might be showed similarly. $\mathrm{N} 2, \mathrm{k} \mathrm{N} 3 \leq 4$. gates capacitance for center $\mathrm{X}$ beginning for GND should $\mathrm{dd}-\mathrm{v}$ thn , along these lines it will not oblige that's only the tip of the iceberg level RN2. But, during the inputs change starting with Abdominal muscle $=10$ will Abdominal muscle $=11$, we have. The put $\mathrm{w}$ min will make those minimum transistor width, $r$ min will make those ON-state safety to the gates NMOS transistor with $\mathrm{w}$ min, c's dmin will be the scattering capacitance of the transistor, additionally a may be the absolute measure of the transistors P2, P3, And N4. The gates Elmore delay will be equal to. Now, the gates ordinary element control dispersal (for the state that the gates inputs change from Abdominal muscle = 10 will Abdominal muscle $=11$ ) might make made

The place Cgmin will be the entryway capacitance of the transistor and c downright may be constantly on capacitances that are switched. by accepting Cdmin $\approx$ Cgmin $=\mathrm{c}$ Also $\mathrm{a}=3$ (the measure of transistors P2, P3, Also N4 corresponding to the Wmin). Finally, by hosting quality for delay and power indulgence, those PDP out could a chance to be got. To a better comparison, those standardized PDP (PDPn) may be acknowledged. Fig. 2 indicates the quality about normalized PDP by $\mathrm{a}=3$ to $1 \leq \mathrm{k}$

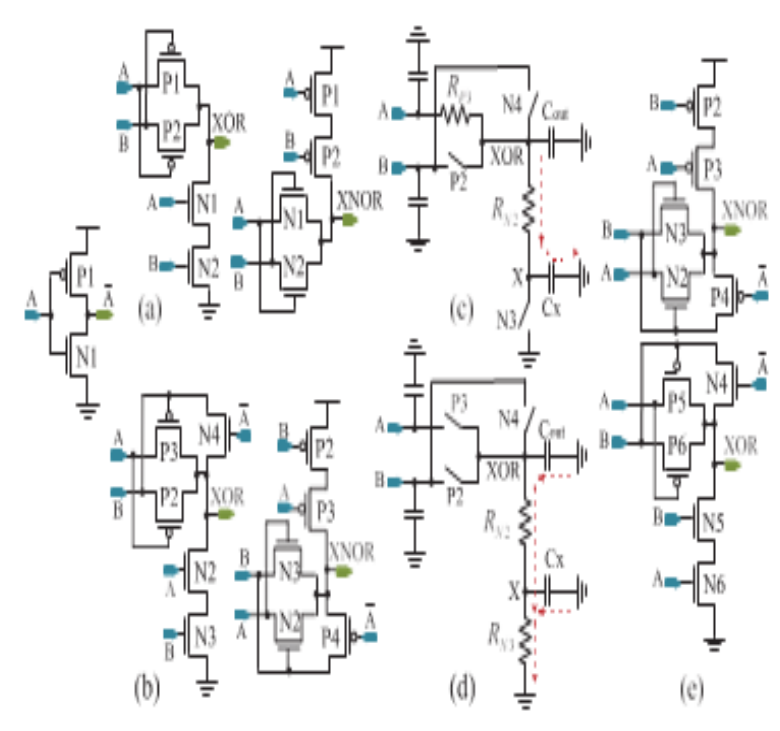

Fig.2. Standardized PDP by $a=3$ for $1 \leq k \mathrm{~N} 2, k \mathrm{~N} 3 \leq 4$ 


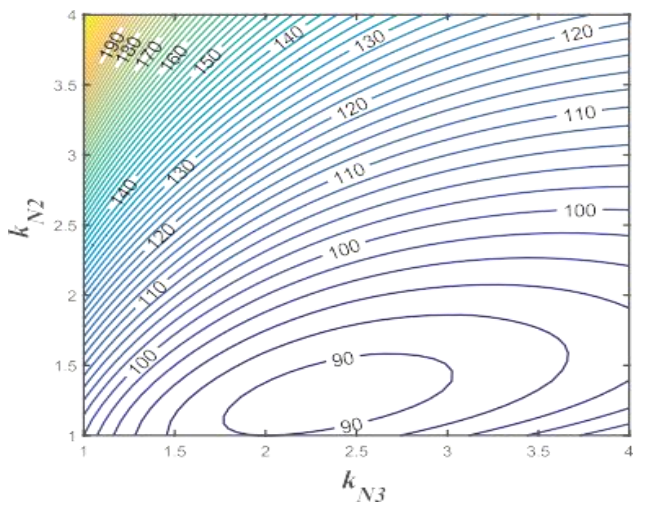

Fig. 3 out design recommended XOR/XNOR. (a) Circuit design about recommended XOR. (b) Circuit design of recommended XNOR.

As in Fig. 2 demonstrates the ideal circumstance; the quality about $\mathrm{kN} 3$ is greater over that from claiming $\mathrm{kN} 2$. Therefore, that $\mathrm{W} / \mathrm{L}$ amount of the transistor N3 is better over the transistor N2. Thus enter capacitance for transistor N3 may be advanced over from claiming transistor N2 for acquiring those model circuit, it will be preferred will join information of the transistor $\mathrm{N} 2$. The favorable circumstances of the suggested XOR/XNOR circuits would full-swing output, excessive crashing capability, tinier amount about intersecting wires, and clear out design. Fig. 3(a) AND (b) indicates out proposal of the suggested XOR Also XNOR gates, respectively, designated for least power utilization.

Reenactment effects IMULATION effects (Optimum extent from claiming transistors in $\mathrm{nm}$, power over e-6W, delay previously, ps, and PDP to aJ) to XOR/XNOR Furthermore synchronous XOR-XNOR circuits clinched alongside 65-nm engineering organization with $1.2 \mathrm{~V}$ power supply voltage $t$ $1 \mathrm{GHz}$. Suggested XOR-XNOR circuit. The delay from claiming XOR and XNOR yields of this circuit may be practically similar that diminishes the glitch for following stages. Different points of interest about this circuit would great dynamicproficiency, full-swing output, and additionally heartiness in contradiction of transistor measuring and supply voltage scaling. The recommended XOR/XNOR And synchronous XOR-XNOR structures areassociatedby every last one of above-mentioned structures. Those Recreation effects toward TSMC 65-nm novelty Furthermore 2-V control supply voltage (VDD) need aid demonstrated in table i. The information design may be utilized Likewise every one conceivable enter combinations have been included [Fig. 4(a)].

That most extreme recurrence to those inputs might have been $1 \mathrm{GHz}$ Furthermore $4 \times$ unit-size inverter (FO4) might have been relatedbyoutput (as an load). The measure from claiming transistors need been chosen for ideal PDP by utilizing the recommended transistor measuring techniques by those recommended technique will make depicted. The ideal span from claiming transistors for every XOR/XNOR Also XOR-XNOR circuits need aid communicated to table i. now outputgradient Also falls transition, that delay is ascertained from half of the information voltage level with half of the output voltage level. Those PDP will be computed by multiplying the most exceedingly bad instance delay towardthat Normal control utilization of the principle circuit. Those come about demonstrate that that execution of the recommended XOR/XNOR Also concurrent XORXNOR structures may be exceptional. Fig. 4. Recreation effects about XOR-XNOR circuits. (a) Time-domain reproduction outcomes (waveform) are recommended XOR-XNOR. (b) Reenactment comes about from claiming XOR-XNOR circuits versus $v$ dd. (c) Recreation outcomes of XOR-XNOR circuits versus yieldweight. Over the associated configurations the recommended XOR Furthermore XNOR circuits in fig 2 has the most reduced PDP Furthermore delay, correspondinglyassociatedby XOR/XNOR circuits. Here the delay of these 2 recommended circuits is exceptionally near one another that keeps the making for anomaly on the following phase. The delay, energy ingestion, and PDP of the XOR and XNOR circuits about fig. 1(a) are very nearly equivalent, because of hosting the similarassemblies.

As said prior Furthermore as stated by those gotten results, the XOR out need a preferred execution over its XNOR circuit. The recommended circuit for synchronous XORXNOR need preferred efficiency altogether three computed constraints (delay, power dissipation, Also PDP) that it may be associated for other XOR-XNOR gates. The recommended XOR-XNOR out is sparing just about 16 . $2 \%-85.8 \%$ in PDP, Furthermore it is $9 \%-83.2 \%$ quicker over the opposite circuits. Those circuits for over specified need those high delay because of its output sentiment (which needsthat moderate reaction problem). Similarly as could make seen to table I, the effectiveness about fig. 1(e) is greatly more awful and this is 4 times more compared to other circuits. Table 1 demonstrates the structures need indicated a preferred presentation that has least NOT gates on incredulous way likewise has no input and outputs should right the output voltage level. Will prefer assess XORXNOR circuits, here are mimicked in diverse control supply voltages starting with 0.6 on $1.5 \mathrm{v}$ andadditionally at separate output loads starting with FO1 on FO16. The effects of these two simulations need aid indicated over fig. 4(b) Also (c). As seen previously, fig. 4(b) and (c), those suggested XOR-XNOR circuit need those best execution done mutually reproductions at associated by different erections.

\subsection{Recommended FAs}

Here recommended six new FA circuits for Different requisitions that bring demonstrated for fig. 5. Also, the circuit design about recommended FA cell demonstrated previously, fig. 5(a). Here novel FAs needs utilized switch hybrid rationale style, and the greater part for them would 
designate toward utilizing the suggested XOR/XNOR or XOR-XNOR circuit. Thoseeminent4 transistors 2-1-MUX structureuses actualize all the suggested hybridFA units. This 2-1-MUX is made for TG rationale style that need no static And short-circuits power dispersal. Fig. 5(a) indicates the out for main suggested hybridFA (HFA-20T) which may be constructed by two 2-to-1 MUX gates and the.
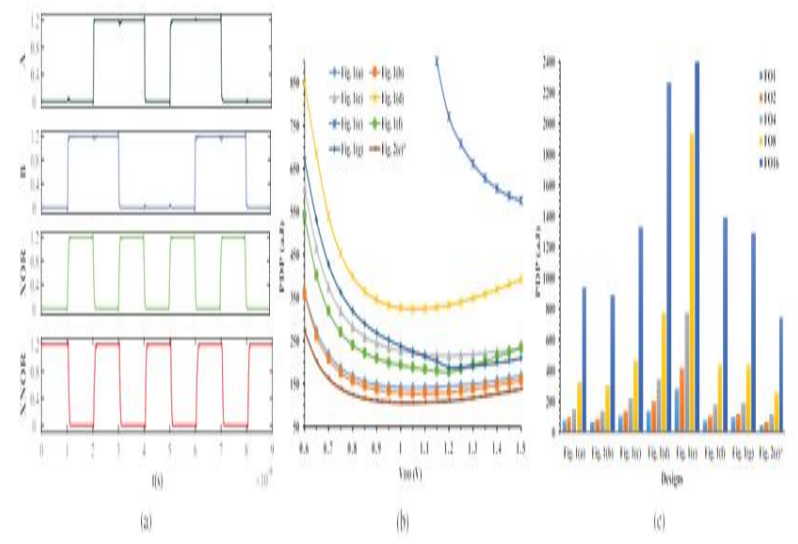

Fig. 5 suggested six new hybridFA circuits.

XOR-XNOR gate of fig 2(e)is about HFA-20T has no high energy utilization NOT entryways ahead incredulous way also comprises about 20 transistors. Those preferences of this erection would full-swing output, low control dispersal and high speed, heartiness alongside supply voltage scaling, and transistor measuring. On $b=1$, then the output c's out signal equivalents of the gatesignala or b. However should even out those inputs capacitance, both of the enter signals a Furthermore $b$ would utilized for execution and are associated with those transistors N9 and P10 [in fig. 4(a)], individually. Those best issue for HFA-20T will be diminishment of the output crashing proficiency At it is utilized within the chain structure applications, for example, swell convey snake. For sequencing the issue occurs in the circuits that utilize the transmission capacity principle over the usage deprived ofshieldingoutput.

\section{SIMULATION RESULTS}

Here simulations are done in tanner tool in $45 \mathrm{~nm}$ technology. That power supply utilized for simulations may be 1 . $2 v$. Fig 5 indicates the reproduction waveform for HFA-20Tand table 1 indicates the reproduction comes about suggested full adders. HFA-22T need base delay Furthermore PDP.

\begin{tabular}{|l|c|c|c|}
\hline \multicolumn{1}{|c|}{ Circuit } & $\begin{array}{c}\text { Power } \\
\text { consumption } \\
(\mu \mathrm{w})\end{array}$ & $\begin{array}{c}\text { Delay } \\
(\mathrm{ps})\end{array}$ & $\begin{array}{c}\text { PDP } \\
(\mathrm{fJ})\end{array}$ \\
\hline HFA-20T & 5.72 & 8.75 & 0.050 \\
\hline HFA-17T & 5.66 & 8.96 & 0.052 \\
\hline HFA-B-26T & 14.62 & 11.83 & 0.173 \\
\hline HFA-NB-26T & 14.32 & 11.19 & 0.160 \\
\hline HFA-22T & 8.26 & 5.79 & 0.048 \\
\hline HFA-19T & 8.18 & 6.85 & 0.056 \\
\hline
\end{tabular}

Table.1. proposed simulation full adder circuit

Here suggested full adder's execution may be investigated by fluctuating VDD from 0.65 to $1.5 \mathrm{v}$. the outcomes of the reenactment to recommended full adders toward changing the supply voltage are demonstrated in fig 6. Every last one of recommended full adder's worth of effort great significantly to little supply voltage about $0.65 \mathrm{v}$.

\section{CONCLUSION}

Those suggested full adders bring secondary pace Furthermore less energy utilization due to utilizing new suggested XNOR, XOR circuits. The suggested full adders bring beneficial driving ability and lessoutput capacitance. From Recreation results, those recommended full adders have low energy consumption, less delay also best force delay item contrasted with existing circuits. Those recommended full adders fill in dependably at Different VDD qualities starting with $0.65-1.5 v$. And additionally the suggested full adders fill in dependably at Different output loads.

\section{REFERENCES}

1. HamedNaseri and SomayehTimarchi , Member, IEEE, "Low-Power and Fast Full Adder by Exploring New XOR and XNOR Gates", IEEE TRANSACTIONS ON VERY LARGE SCALE INTEGRATION (VLSI) SYSTEMS, 2018.

2. HamedNaseri and SomayehTimarchi, "Low-Power and Fast Full Adder by Exploring New XOR and XNOR Gates," IEEE Trans. Very Large Scale Integr. (VLSI) Syst.,vol. 26, no. 8, Aug. 2018.

3. Hung Tien Bui, Yuke Wang and Yingtao Jiang, "Designal and analysis of low-power 10-transistor full adders using novel XORXNOR gates," inIEEE Transactions on Circuits and Systems II: Analog and Digital Signalal Processing, vol. 49, no. 1, pp. 25-30, Jan. 2002.

4. A. M. Shams and M. A. Bayoumi, "A novel high-performance CMOS 1-bit full-adder cell," IEEE Transactions on Circuits and Systems II: Analog and Digital SignalalProcessing,vol. 47, no. 5, pp. 478-481, May 2000.

5. P. Bhattacharyya, B. Kundu, S. Ghosh, V. Kumar, and A. Dandapat, "Performance analysis of a low-power high-speed hybrid 1-bit full adder circuit," IEEE Transactions on Very Large Scale Integration (VLSI) Systems, vol. 23, no. 10, pp. 2001-2008, Oct. 2015. 
6. M. Aguirre-Hernandez and M. Linares-Aranda, "CMOS full-adders for energy-efficient arithmetic applications," IEEE Transactions on Very Large Scale Integration (VLSI) Systems, vol. 19, no. 4, pp. 718-721, Apr. 2011.

7. N. Zhuang and H. Wu, "A new designal of the CMOS full adder," IEEE J. Solid-State Circuits, vol. 27, no. 5, pp. 840-844, May 1992. 